\title{
Diabetes Mellitus Tipo 1: Perfil Glicêmico e Consumo Alimentar em um Ambulatório de Nutrição Pediátrica
}

\author{
Thais Marini da Rosa ${ }^{1}$, Sandra Costa Valle ${ }^{2}$, Camila Irigonhé Ramos ${ }^{3}$
}

\begin{abstract}
RESUMO
Introdução: os pacientes pediátricos com Diabetes Mellitus tipo 1 (DM1) são vulneráveis a um mau controle metabólico ante o crescimento físico intensificado e a maturação sexual, que alteram suas respostas fisiológicas. O controle glicêmico, no entanto, é um objetivo fundamental e constante para estes pacientes, sendo a dieta um importante fator nesse processo. Objetivo: investigar o perfil glicêmico e o consumo alimentar de crianças e adolescentes assistidos de um ambulatório de nutrição pediátrica do Sistema Único de Saúde (SUS) do Sul do Brasil. Métodos: estudo longitudinal, com dados de controle glicêmico coletados dos prontuários de saúde na primeira consulta nutricional (inicial) e após 10 meses de assistência nutricional (final). O perfil glicêmico foi avaliado por meio das glicemias capilares $\left(\mathrm{mg} \mathrm{dL}^{-1}\right)$ pré e pós-prandiais, da hemoglobina glicada (\%) e da glicemia média estimada. O consumo alimentar foi avaliado após dois recordatórios de 24 horas aplicados durante a semana, incluindo a segunda-feira. Resultados: os parâmetros de controle glicêmico mostraram melhora na consulta final, em especial com adequação da hemoglobina glicada (\%). O consumo dos principais grupos alimentares fonte de carboidratos, ficou adequado às recomendações vigentes. $O$ consumo de frutas, legumes e verduras, entretanto, foi insuficiente e o das carnes foi excessivo. Conclusão: o perfil glicêmico e o consumo de carboidratos caracterizaram-se por adequação após assistência nutricional. Em particular, a hemoglobina glicada \%, o número e o tamanho das porções de alimentos fonte de carboidratos, atenderam às recomendações para o controle glicêmico de crianças e adolescentes com DM1.
\end{abstract}

Palavras-chave: Diabetes mellitus tipo 1. Glicemia. Consumo alimentar. Crianças. Adolescentes. Alimentação.

DIABETES MELLITUS TYPE 1: GLYCEMIC PROFILE AND FOOD INTAKE AT A PEDIATRIC NUTRITION OUTPATIENT CLINIC ABSTRACT

Introduction: Pediatric patients with Type 1 Diabetes Mellitus (DM1) are vulnerable to metabolic control of intensified physical growth and sexual maturation that alter their physiological responses. However, glycemic control is fundamental and constant goal for these patients, being the diet an important factor in this process. Objective: to investigate the glycemic profile and dietary intake of children and adolescents assisted from a pediatric nutrition outpatient clinic of the Unified Health System (SUS) in Southern Brazil. Methods: longitudinal study, with glycemic control data collected in health records, first nutritional consultation (initial) and after 10 months of nutritional assistance (final). The glycemic profile was evaluated by means of pre and postprandial capillary glycemia $\left(\mathrm{mg} \mathrm{dL}^{-1}\right)$, glycated hemoglobin (\%) and estimated mean glycemia. Food consumption assessed using two 24-hour records during the week, including Monday. Results: glycemic control parameters were improved in the final consultation, especially with the adequacy of glycated hemoglobin (\%). The consumption of the main carbohydrate food groups was adequate by the current recommendations. However, consumption of fruits and vegetables was insufficient and the meat was excessive. Conclusion: After nutritional assistance, the glycemic profile and the carbohydrate intake were, characterize by adequacy. In particular, glycated hemoglobin\%, number and size of carbohydrate source foods met the recommendations for glycemic control of children and adolescents with DM1.

Keywords: Diabetes mellitus type 1. Glycemia. Food consumption. Children. Adolescents. Diet.

RECEBIDO EM: 24/4/2019

MODIFICAÇÕES REQUERIDAS EM: 15/7/2019

ACEITO EM: 18/9/2019

\footnotetext{
Graduanda de Nutrição (Universidade Federal de Pelotas - Ufpel). Monitora voluntária no projeto de Ensino em Genética do Metabolismo. Bolsista no Projeto de Extensão (2017), prestando assistência clínica nutricional a crianças e adolescentes com Diabetes Mellitus e colaboradora do Projeto de Extensão a crianças atendidas no Centro de Atendimento ao Autista, como voluntária desenvolvendo ações de vigilância alimentar e nutricional e orientações nutricionais a crianças com autismo e responsáveis. thr.marini@gmail.com

Professora adjunta, nível II, da Universidade Federal de Pelotas. Mestre em Ciências Biológicas (Bioquímica) e doutora em Ciências Biológicas (Fisiologia) pela Universidade Federal do Rio Grande do Sul. sandracostavalle@gmail.com

${ }^{3}$ Nutricionista, doutoranda no PPG em Enfermagem da Universidade Federal de Pelotas - UFPel. Mestra em Nutrição e Alimentos e especialista em Saúde Pública, Saúde da Família e Comunidade e em Nutrição em Neuropsiquiatria.mila85@gmail.com
} 


\section{INTRODUÇÃO}

O DM1 é uma condição crônica caracterizada pela destruição autoimune das células $\beta$ pancreáticas, provocando a deficiência na produção de insulina. Essa condição pode se estabelecer em indivíduos de todas as idades, mas geralmente acomete crianças e adolescentes. Quando detectada em fase inicial do desenvolvimento, a hiperglicemia prolongada pode comprometer precocemente a qualidade de vida, em virtude dos danos vasculares que altas concentrações glicêmicas podem causar (AMERICAN..., 2019).

Nos últimos anos houve um aumento na prevalência de DM1 no Brasil e no mundo. A estimativa mundial corresponde a 542 mil crianças e adolescentes, com idade entre zero e 14 anos, com a doença. Dentre os países com as maiores prevalências, encontram-se Estados Unidos, Índia e Brasil (INTERNATIONAL..., 2015). O Brasil não possui um estudo de base populacional que identifique especificamente os casos de DM1. Estima-se que a prevalência e a incidência da doença no país, em indivíduos menores de 14 anos, sejam de 4/10 mil e 8/100 mil habitantes, respectivamente (TELES; FORNÉS, 2011; SOCIEDADE..., 2017a).

A terapêutica do DM1 na criança e no adolescente prevê o controle metabólico, alimentação adequada e prática regular de atividade física (DONG et al., 2017; AMERICAN..., 2019). O principal objetivo do tratamento do DM1 é a obtenção de um bom controle metabólico, necessário para o crescimento e desenvolvimento adequado e a prevenção de complicações agudas e crônicas (SOCIEDADE..., 2017a; AMERICAN..., 2019). $\mathrm{O}$ paciente pediátrico com DM1, entretanto, difere-se do adulto quanto a suas respostas fisiológicas ante o tratamento, em razão do crescimento físico intensificado e a maturação sexual (AMERICAN..., 2019).

De acordo com o Diabetes Control and Complications Trial (DCCT), o controle glicêmico pode retardar o início e a progressão de complicações micro e macrovasculares do DM1 (INTERNATIONAL..., 2015). Uma das principais recomendações desse estudo foi que no DM1 o planejamento dietético deve ser individualizado, permitir flexibilidade nas escolhas alimentares e, sobretudo, possibilitar um controle metabólico adequado (AMERICAN..., 2019).

Se, por um lado, é necessário atender estas recomendações, por outro os pacientes com DM1 constituem um grupo especialmente vulnerável. Salienta-se que, além do diabetes, as necessidades nutricionais estão aumentadas, o padrão alimentar e estilo de vida estão em constante mudança e há uma suscetibilidade às influências ambientais, em particular nos adolescentes (WYLIE-ROSETT et al., 2012; DONG et al., 2017). Acredita-se, portanto, que o monitoramento do consumo alimentar em pacientes com DM1 é importante para o ajuste da dieta e para um melhor controle metabólico. Além disso, permite a identificação de um comportamento de risco, a fim de garantir o pleno potencial de crescimento e desenvolvimento e prevenir complicações da doença (WYLIE-ROSETT et al., 2012; SOCIEDADE..., 2017a).

Diante do exposto, o objetivo do presente estudo foi investigar o perfil glicêmico e o consumo alimentar de crianças e adolescentes assistidos em um ambulatório de nutrição pediátrica do Sistema Único de Saúde (SUS) do Sul do Brasil.

\section{METODOLOGIA}

Estudo longitudinal realizado com crianças e adolescentes assistidos no ambulatório de nutrição infantil do Centro de Diabetes e Hipertensão da Universidade Federal de Pelotas, Pelotas, Rio Grande do Sul, Brasil. Foram incluídos no estudo crianças e adolescentes, de ambos os sexos, com diagnóstico de diabetes mellitus tipo 1 (DM1), que consultaram no serviço público no período de junho de 2017 a maio de 2018. Foram excluídos aqueles que compareceram apenas na primeira consulta. O protocolo do estudo foi submetido e aprovado pelo Comitê de Ética em Pesquisa da Faculdade de Medicina da Universidade Federal de Pelotas, CAAE número 33883914.4.0000.5317.

Foram coletados dados sociodemográficos, clínicos, antropométricos e de consumo alimentar. Dos prontuários foram obtidas as seguintes informações: idade (anos); sexo (masculino ou feminino); história de doenças na família (sim/não); prática de atividade física programada (sim ou não); data da primeira e da última consulta (dia/mês/ano); número de consultas com nutricionista; peso $(\mathrm{kg})$; estatura $(\mathrm{m})$; hemoglobina glicada (HbA1c, \%); glicemias pré-prandiais (mg.dL$\left.{ }^{1}\right)$ relativas ao café da manhã, almoço e jantar.

O peso foi aferido em balança digital, com capacidade de $150,0 \mathrm{~kg}$, precisão de $100 \mathrm{~g}$. A estatura foi aferida com uso de estadiômetro acoplado à balança $(2,0 \mathrm{~m}$, precisão de $0,1 \mathrm{~cm})$. Estas medidas foram obtidas em duas vezes consecutivas, sendo a média de cada variável utilizada para cálculo do índice de massa corporal-IMC (peso $(\mathrm{kg}) /$ estatura $\left(\mathrm{m}^{2}\right)$ ). A glicemia média estimada foi calculada segundo a equação: $28,7 \mathrm{x}$ HbA1c-46,7 (SOCIEDADE..., 2017b). As glicemias pré-prandiais e $\mathrm{HbA} 1 \mathrm{c}$ foram obtidas na consulta inicial e final. Esta última aconteceu, aproximadamente, dez meses após a primeira. 
O diagnóstico nutricional antropométrico foi obtido segundo o escore-z do IMC/idade, utilizando-se como referência os pontos de corte da Organização Mundial da Saúde (OMS), em 2006 e 2007.

Na primeira consulta de nutrição foi realizada anamnese detalhada, identificados sinais cardinais de má controle metabólico, o registro glicêmico diário e o esquema de insulinização empregado e quem o realiza. Foram investigadas a presença de alergias e/ou intolerâncias alimentares, a ingestão hídrica, o hábito intestinal, o consumo de energia e nutrientes e sua adequação diante das necessidades. A intervenção nutricional previa o estabelecimento de uma meta glicêmica, o cálculo das necessidades de energia, a recomendação de micronutrientes e um plano de alimentação elaborado e orientado na perspectiva da contagem de carboidratos (AMERICAN..., 2018). Ainda o paciente e seus familiares foram orientados quanto à identificação dos alimentos fonte de carboidratos e seus teores em porções usuais de consumo. Além disso, receberam um treinamento sobre medidas caseiras, priorizando o uso de utensílios próprios. Ao final das consultas os pacientes realizavam a leitura dos rótulos de alimentos que desejavam consumir, mas ainda tinham dúvidas sobre sua pertinência dietética.

O consumo alimentar foi avaliado após a realização de, no mínimo, dois recordatórios de 24 horas (R24h), aplicados durante a semana, incluindo a segunda-feira. Os R24h foram respondidos pelos pais ou responsáveis, os quais foram orientados a falar sobre horário e tipo de refeição, variações dos alimentos e quantidades. Para auxiliar na identificação do tamanho das porções dos alimentos consumidos, foi utilizado um guia fotográfico com porções usualmente consumidas no Brasil (MONEGO et al., 2013).

Os alimentos referidos nos R24h foram listados e classificados segundo os grupos da Pirâmide Alimentar Brasileira (BRASIL, 2006), obtendo-se, assim, a frequência observada (FO) de cada grupo. Já a frequência esperada (FE) foi estabelecida, multiplicando-se o número total de R24h (12) pelo número de porções recomendadas para cada grupo alimentar. $\mathrm{O}$ número de porções foi estabelecido com base na média da necessidade de energia da amostra, que correspondeu a $1.850 \mathrm{kcal} / \mathrm{dia}$ (BRASIL, 2006). Para verificar a adequação do consumo alimentar, segundo os grupos da Pirâmide, calculou-se a frequência obtida a partir da razão entre FO/FE. Para o cálculo da necessidade energética, utilizou-se os critérios fornecidos pela Food and Agriculture, of United States of America, de acordo com o sexo e a idade (ACCIOLY, 2012).
Os resultados são apresentados como frequência absoluta e relativa, média \pm desvio padrão (dp). A comparação entre os períodos das consultas foi realizada por meio do teste $t$ de Student, para amostras repetidas. Já o teste t Student para uma amostra foi utilizado para testar o valor de hemoglobina glicada da primeira ou da última consulta com o valor de 7,5\%, recomendado pela SBD. Para as análises utilizou-se o software BioStat 5.0 e o erro $\alpha$ aceitável foi estabelecido em $5 \%$.

\section{RESULTADOS}

No ambulatório de nutrição pediátrica havia um total de oito pacientes assistidos por alteração glicêmica e, dentre estes, seis apresentavam diagnóstico de DM1. Em razão da falta de dados no prontuário de saúde ocorreu uma perda de acompanhamento. No total, cinco pacientes $(83,3 \%)$ com DM1 foram incluídos no estudo, e não houve recusas à participação neste. As características de pacientes com DM1 assistidos no ambulatório de Nutrição pediátrica do Centro de Diabetes e Hipertensão, estão descritas na Tabela 1.

Tabela 1 - Características de pacientes com diabetes mellitus tipo 1 assistidos no ambulatório de Nutrição pediátrica do Centro de Diabetes e Hipertensão, Pelotas-RS, 2018. $\mathrm{N}=5$

\begin{tabular}{lcc}
\hline Variáveis & & \\
\hline Idade (anos) $^{*}$ & 11,5 & 2,2 \\
Sexo (M/F) & $4 / 1$ & $80,0 / 20,0$ \\
Histórico de Doença na Família $^{* *}$ (Sim/Não) & $1 / 4$ & $20,0 / 80,0$ \\
Atividade Física Programada** (Sim/Não) $^{* *}$ & $4 / 1$ & $80,0 / 20,0$ \\
Acompanhamento Dietético (meses) $^{*}$ & 10,9 & 0,7 \\
Número de Consultas* $^{*}$ & 5 & 2 \\
Tempo Entre Consultas (meses) $^{*}$ & 2,3 & 0,6 \\
Peso (kg) $^{*}$ & 46,7 & 12,5 \\
Estatura (m) $^{*}$ & 1,5 & 0,2 \\
IMC (kg/m $\left.^{*}\right)^{*}$ & 18,9 & 2,5 \\
\hline
\end{tabular}

${ }^{* *}$ Variável apresentada como frequência absoluta e relativa. *Variável apresentada como média $\pm \mathrm{dp}$.

Na Tabela 2 mostra-se as características de controle glicêmico de pacientes DM1 de acordo com o tempo de acompanhamento nutricional. As glicemias que precederam o café da manhã, almoço e jantar foram, respectivamente, $151,4 \pm 39,7 \mathrm{mg}^{\mathrm{dL}} \mathrm{dL}^{-1}, 151,4 \pm$ $55,9 \mathrm{mg} \cdot \mathrm{dL}^{-1}$ e $198,8 \pm 56,2 \mathrm{mg} \cdot \mathrm{dL}^{-1}$. A glicemia pré-prandial do jantar foi estatisticamente menor ( $p \leq$ $0,05)$ na consulta final, quando comparada à inicial $\left(198,8 \pm 56,2 \mathrm{mg} \cdot \mathrm{dL}^{-1}\right.$ vs $\left.141,0 \pm 59,1 \mathrm{mg} \cdot \mathrm{dL}^{-1}\right)$. 
Apesar de não observarmos diferença estatística significativa, a $\mathrm{HbA1c}(\%)$ foi, aproximadamente, $25 \%$ menor $(p>0,05)$ na consulta final comparada à inicial $(9,8 \pm 2,3$ vs $7,3 \%)$. Do mesmo modo, a glicemia média estimada foi, aproximadamente, 30,5\% (71,7 $\left.\mathrm{mg} \cdot \mathrm{dL}^{-1}\right)$ menor na última consulta $(p>0,05)$, quando comparada à estimada no período da primeira $(234,5$ $\pm 2,3 \mathrm{mg} \cdot \mathrm{dL}^{-1}$ vs $\left.162,81 \pm-15,1 \mathrm{mg} \cdot \mathrm{dL}^{-1}\right)$. Ainda, na primeira consulta, a HbA1c ficou $25 \%$ acima $(p>0,05)$ do valor limítrofe no DM1 (9,8\% vs 7,5\%). Na última consulta, porém, mostrou-se adequada e abaixo deste limite $(7,3 \%$ vs $7,5 \%$; resultados não apresentados em Tabelas e Figuras).

Tabela 2 - Características de controle glicêmico de pacientes com diabetes mellitus tipo 1 assistidos no ambulatório de Nutrição pediátrica do Centro de Diabetes e Hipertensão, Pelotas-RS, 2018. N=5

\begin{tabular}{|c|c|c|c|c|c|}
\hline & \multicolumn{4}{|c|}{ Consulta } & \multirow[b]{3}{*}{$p$} \\
\hline & \multicolumn{2}{|c|}{ Inicial } & \multicolumn{2}{|c|}{ Final } & \\
\hline & Média & Dp & Média & Dp & \\
\hline \multicolumn{6}{|l|}{$\begin{array}{l}\text { Glicemia Pré-prandial } \\
\left(\mathrm{mg}^{\mathrm{dL}} \mathrm{L}^{-1}\right)\end{array}$} \\
\hline Café da manhã & 151,4 & 39,7 & 163,8 & 83,1 & 0,715 \\
\hline Almoço & 151,4 & 55,9 & 159,2 & 29,4 & 0,840 \\
\hline Jantar & 198,8 & 56,2 & 141,0 & 59,1 & $0,050^{*}$ \\
\hline Hemoglobina glicada (\%) & 9,8 & 2,3 & 7,3 & 1,1 & 0,174 \\
\hline $\begin{array}{l}\text { Glicemia Média } \\
\text { Estimada (mg.dL-1) }\end{array}$ & 234,6 & 19,3 & 162,8 & $-15,1$ & 0,174 \\
\hline
\end{tabular}

A comparação entre a frequência de consumo alimentar observada (FO) e a frequência esperada $(\mathrm{FE})$, realizada pela razão $\mathrm{FO} / \mathrm{FE}$, mostrou um consumo do grupo das carnes 3,9 vezes acima do esperado. Por outro lado, o consumo do grupo das frutas foi 0,3 e das verduras e legumes foi 0,1 em relação ao esperado (Tabela 3).
Ao transformar-se a frequência obtida para cada grupo de alimentos em porções da Pirâmide Alimentar, foi possível constatar uma mudança na posição dos grupos, pois, em razão do baixo consumo de frutas, verduras e legumes, estes ficaram no ápice. 0 grupo das carnes, entretanto, ficou no segundo nível da Pirâmide por seu consumo elevado (Figura 1).

Figura 1 - Pirâmide alimentar a partir dos recordatórios de $24 \mathrm{~h}$ de pacientes com diabetes mellitus tipo 1 , assistidos no ambulatório de Nutrição pediátrica, do Centro de Diabetes e Hipertensão, Pelotas-RS, 2018. N=5.

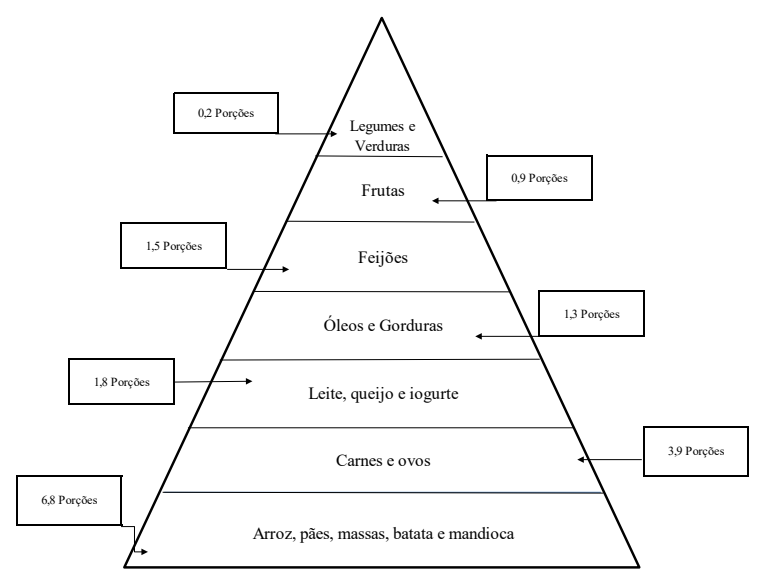

\section{DISCUSSÃO}

Neste estudo verificou-se que o consumo dos principais grupos de alimentos fonte de carboidratos foi adequado às recomendações vigentes após o início do acompanhamento nutricional de pacientes com DM1. Apesar disso, o consumo de frutas, legumes e verduras foi insuficiente e do grupo das carnes foi excessivo. Ainda, constatou-se melhora no perfil glicêmico pré-prandial do jantar. Apesar, no entanto,

Tabela 3 - Frequência obtida, segundo os grupos da Pirâmide Alimentar Brasileira, da dieta de pacientes com diabetes mellitus tipo 1 assistidos no ambulatório de Nutrição pediátrica do Centro de Diabetes e Hipertensão, Pelotas-RS, 2018. $\mathrm{N}=5$

\begin{tabular}{|c|c|c|c|c|c|c|c|}
\hline & \multicolumn{3}{|c|}{ Frequência Esperada } & \multicolumn{3}{|c|}{ Frequência Observada } & \multirow{2}{*}{$\begin{array}{c}\text { Frequência Obtida } \\
\mathrm{FO} / \mathrm{FE}^{*}\end{array}$} \\
\hline & Total & Diária & kcal & Total & Diária & kcal & \\
\hline $\begin{array}{l}\text { Arroz, pães, massas, batata } \\
\text { e mandioca }\end{array}$ & 72,0 & 6,0 & 900,0 & 81,0 & 6,8 & 1012,5 & 1,1 \\
\hline Verduras e legumes & 36,0 & 3,0 & 135,0 & 2,0 & 0,2 & 7,5 & 0,1 \\
\hline Frutas & 36,0 & 2,0 & 140,0 & 11,0 & 0,9 & 64,2 & 0,3 \\
\hline Leite, queijo e iogurte & 36,0 & 3,0 & 360,0 & 21,0 & 1,8 & 210,0 & 0,6 \\
\hline Carnes e ovos & 12,0 & 1,0 & 190,0 & 46,5 & 3,9 & 736,3 & 3,9 \\
\hline Feijões & 12,0 & 1,0 & 55,0 & 17,5 & 1,5 & 80,2 & 1,5 \\
\hline Óleos e gorduras & 12,0 & 1,0 & 73,0 & 15,5 & 1,3 & 94,3 & 1,3 \\
\hline Açúcares e doces & 0,0 & 0,0 & 0,0 & 1,0 & 0,1 & 11,0 & 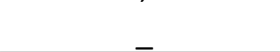 \\
\hline
\end{tabular}

${ }^{*} \mathrm{FO} / \mathrm{FE}=$ Frequência observada/Frequência Esperada 
da significativa melhora dos demais parâmetros glicêmicos, em particular da $\mathrm{HbA} 1 \mathrm{c}$, o reduzido tamanho da amostra pode ter limitado a detecção de diferença estatística neste caso.

O objetivo principal do tratamento do DM1 é a obtenção do controle metabólico, necessário para um crescimento e desenvolvimento adequado na infância e na adolescência. As recomendações terapêuticas para o controle do DM1 baseiam-se na reposição insulínica, adequação alimentar e prática de atividade física, minimizando os riscos de complicações agudas e crônicas (AMERICAN..., 2019). No ambulatório de nutrição pediátrica deste estudo, a assistência tem como base as recomendações da Sociedade Brasileira de Diabetes, que objetivam o controle metabólico e a autonomia dos pacientes para escolhas alimentares nutritivas e saudáveis.

As metas de controle glicêmico, a exemplo da $\mathrm{HbA} 1 \mathrm{c} \%$, em crianças e adolescentes, é $\mathrm{HbA} 1 \mathrm{c}<7,5$ (AMERICAN..., 2019). Neste estudo, a concentração de $\mathrm{HbA} 1 \mathrm{c}$ reduziu em média $25 \%$ ao final do acompanhamento, ficando abaixo de 7,5\%. Apesar da ausência de diferença estatística significativa entre os períodos analisados, a adequação da $\mathrm{HbA} 1 \mathrm{c}$ representa um importante fator de prevenção a agravos à saúde do DM1 no jovem, em especial, ao considerarmos o aumento exponencial do risco de complicações vasculares associado à concentração de $\mathrm{HbA} 1 \mathrm{c} \geq 7,5 \%$ (SOCIEDADE..., 2017c).

É importe salientar que $80 \%(n=4)$ dos pacientes deste estudo estavam na puberdade, momento no qual os hormônios contrarregulatórios à ação da insulina contribuem para a hiperglicemia e mau controle do DM1. Por outro lado, observou-se que a maioria $(80 \%)$ dos pacientes praticava atividade física programada, fato que pode melhorar a glicemia média estimada (GME) a longo prazo. $O$ exercício físico é indicado para o DM1 por trazer benefícios no controle metabólico, diminuição do risco cardiovascular e prevenção de complicações do diabetes (LIMA et al., 2017).

A alimentação saudável preconizada para o DM1 corresponde à mesma destinada a crianças e adolescentes hígidos. O plano alimentar para o DM1 deve estar de acordo com as recomendações de energia e nutrientes, específicos para cada faixa etária e sexo, e proporcionar flexibilidade às atividades diárias (DONG et al., 2017). Especialmente em relação à quantidade de carboidratos, entretanto, a adequação do consumo alimentar ao esquema de insulinização é essencial para um bom controle glicêmico no DM1 (WYLIE-ROSETT et al., 2012; DONG et al., 2017).
Um aspecto positivo destacado na presente pesquisa foi o consumo adequado de alimentos fonte de açúcares, a exemplo do grupo dos pães, arroz, massas e batatas. $O$ número de porções obtidas destes alimentos ficou apenas $10 \%$ acima do esperado, considerando-se a média do VET, de acordo com a idade e sexo. Ainda, o consumo de alimentos fonte de açúcares simples, como o açúcar de mesa e alimentos à base/contendo este, não foi observado. Este resultado pode ter dois aspectos relacionados. $O$ primeiro a chamada fase de "lua de mel", quando, após o diagnóstico, a criança e seus familiares buscam o controle constante de carboidratos ingeridos, na intenção de evitar a presença de sintomas associados ao momento do diagnóstico (ALBUQUERQUE et al., 2014; DONG et al., 2017; AMERICAN..., 2019). Esta fase esteve presente para $60 \%(n=3)$ dos pacientes, uma vez que a consulta no ambulatório correspondeu a primeira consulta após o diagnóstico e alta hospitalar. Já o segundo aspecto diz respeito ao esforço da equipe de saúde no treinamento dos pacientes e de seus familiares, na identificação de alimentos fonte de açúcares e na importância do controle quantitativo destes alimentos ante o esquema de insulinização (ALBUQUERQUE et al., 2014; DONG et al., 2017; AMERICAN..., 2019).

Apesar do cuidado com as fontes de açúcares, o aumento no consumo do grupo das carnes e ovos sugere uma substituição por estes alimentos. Resultados similares a este já foram observados em outras pesquisas (TELES; FORNÉS, 2011; ALBUQUERQUE et al., 2014; QUEIROZ et al., 2015). Teles e Fornés (2011), ao avaliarem crianças e adolescentes com DM1, verificaram consumo de dieta hiperproteica. Resultados similares quanto ao consumo excessivo de proteínas por indivíduos DM1 também foram observados por Queiroz et al. (2015). Em particular, este pesquisador verificou que o consumo médio de gorduras ultrapassou a recomendação para os pacientes. No atual estudo, o consumo de gorduras também ficou acima do esperado (30\%).

Em relação às proteínas, é importante destacar que, aproximadamente, $60 \%$ do excesso de proteína consumida, o equivalente a uma cota superior a $20 \%$ do VET, transforma-se em glicose, via gliconeogênese. Desta maneira, o aumento no total de proteínas ingeridas contribui para a elevação da glicemia, em geral de forma tardia quanto ao horário de consumo (SOCIEDADE..., 2017a). Segundo as Diretrizes da Sociedade Brasileira de Diabetes (2017-2018), o consumo de 
proteínas deve ser adequado em pacientes com DM1 de maneira a prevenir sobrecarga renal e lesão deste órgão a longo prazo.

No que diz respeito às frutas, legumes e verduras (FL\&V), estudos conduzidos no Brasil convergem quanto à observação de insuficiência destes alimentos nas refeições entre as famílias (LEAL et al., 2010; BRASIL, 2011). O consumo diário recomendado é de três porções de frutas e três porções de legumes e verduras, salientando a importância de diversificar esses alimentos nas refeições (BRASIL, 2006). Na atual pesquisa, o consumo alimentar extremamente baixo de FL\&V destaca-se como um ponto importante a ser melhorado com os pacientes e seus familiares. Além de tudo, as FL\&V podem auxiliar na prevenção de diversas doenças por meio de substâncias protetoras, como vitaminas e minerais antioxidantes, fibras e diferentes componentes fenólicos (BRASIL, 2006). Assim, a ingestão adequada de FL\&V contribui para a manutenção da boa saúde em pacientes DM1 (AMERICAN..., 2019).

Quanto ao grupo dos leites, iogurtes e queijos, o consumo alimentar atingiu $60 \%$ do recomendado, necessitando adequação, especialmente em virtude da faixa etária, cuja recomendação de cálcio é de $1300 \mathrm{mg} \mathrm{dia}^{-1}$ (PADOVANI et al., 2006). Um consumo inadequado de produtos lácteos por adolescentes tem sido verificado em outras pesquisas, mostrando a necessidade de reforçar a indicação de laticínios ou de suplementos de cálcio na contraindicação permanente destes (ALBUQUERQUE et al., 2014; QUEIROZ et al., 2015). Em especial, na adolescência, ocorre a transformação de condrócitos em matriz óssea, incrementando a demanda de cálcio. Além disso, 25\% da densidade mineral óssea do adulto é obtida nesta fase da vida (ACCIOLY et al., 2012).

Um fator limitante deste estudo foi o tamanho da amostra. Os casos de DM1 geralmente são encaminhados para ambulatórios especializados da rede pública e o serviço de Nutrição Pediátrica tem sido atuante neste cenário desde sua introdução em junho de 2017. Neste contexto, por se tratar de uma doença com alta frequência de complicações, considera-se que o estudo contribuiu com informações relevantes sobre os pacientes pesquisados e também subsidia novas condutas a serem adotadas pelos profissionais do serviço.

\section{CONCLUSÃO}

O perfil glicêmico pré-prandial do jantar e o consumo alimentar de carboidratos caracterizaram-se por melhora após assistência nutricional a crianças e adolescentes com DM1. Em especial, o número e o tamanho das porções ingeridas de alimentos fonte de açúcares foram adequadas. Apesar disso, o consumo insuficiente de frutas, legumes e verduras indica a necessidade de estratégias que estimulem mais as famílias e os pacientes ao consumo destes alimentos. O desenvolvimento de oficinas, com receitas simples e palatáveis à base de vegetais, poderia ser uma alternativa para se alcançar a adequação desse consumo.

\section{REFERÊNCIAS}

ACCIOLY, E. et al. Nutrição em obstetrícia e pediatria. 2. ed. rev. atual. Rio de Janeiro: Cultura Médica, 2012. 277p.

ALBUQUERQUE, I. Z. et al. Contagem de carboidratos, estado nutricional e perfil metabólico em adolescentes com diabetes mellitus tipo 1. Scientia Medica, 24(4), p. 343-352, 2014.

AMERICAN DIABETES ASSOCIATION. Children and Adolescents: Standards of Medical Care in Diabetes 2018. Diabetes Care, 41 (1), S126, 2018.

AMERICAN DIABETES ASSOCIATION. Children and Adolescents: Standards of Medical Care in Diabetes 2019. Diabetes Care, 42 (1), S148, 2019.

BRASIL. Ministério da Saúde. Secretaria de Atenção à Saúde. Departamento de Atenção Básica. Guia alimentar para a população brasileira Promovendo a Alimentação Saudável. 2. ed. Brasília: Ministério da Saúde; Secretaria de Atenção à Saúde; Departamento de Atenção Básica, 2006.

BRASIL. Ministério do Planejamento, Orçamento e Gestão. Instituto Brasileiro de Geografia e Estatística - IBGE. Diretoria de Pesquisas Coordenação de Trabalho e Rendimento. Pesquisa de Orçamentos Familiares 2008-2009. Análise do consumo pessoal no Brasil. Rio de Janeiro: Ministério do Planejamento, Orçamento e Gestão, 2011.

DONG, I. J. et al. Influence of nutritional intervention on children with type 1 diabetes mellitus and DPP-4 in serum. Experimental and Therapeutic Medicine, 14, p. 913-916, 2017.

INTERNATIONAL DIABETES FEDERATION. IDF Diabetes Atlas [Internet]. 7. ed. Brussels: International Diabetes Federation, 2015. Available from: https: www.idf.org/diabetes-atlas. Cited: 16 jul. 2018.

LEAL, G. V. S. et al. Consumo alimentar e padrão de refeições de adolescentes. Revista Brasileira de Epidemiologia, São Paulo, v.13, n. 3, p. 457-467, 2010.

LIMA, L. A. P.; WEFFORT, V. R. S.; BORGES, M. de F. Avaliação da qualidade de vida de crianças com diabetes Mellitus tipo 1. Ciência, Cuidado e Saúde, 10(1), p. 127-133, 2011.

LIMA, V. A. et al. Efeito agudo dos exercícios intermitentes sobre a glicemia de adolescentes com diabetes tipo 1. Revista Brasileira de Medicina e Esporte, v. 23, n. 1, p. 12-15, 2017. 
MARQUES, R. M. B.; FORNÉS, N. S.; STRINGHINI, M. L. F. Fatores socioeconômicos, demográficos, nutricionais e de atividade física no controle glicêmico de adolescentes portadores de diabetes melito tipo 1. Arquivos Brasileiros de Endocrinologia \& Metabologia, v. 55, n. 3, p. 194-202, 2011. MONEGO, E. et al. Alimentos brasileiros e suas porções: um guia para avaliação do consumo alimentar. Rio de Janeiro, RJ: Rubio, 2013.

PADOVANI, R. M. et al. Dietary reference intakes: aplicabilidade das tabelas em estudos nutricionais. Revista de Nutrição de Campinas, v. 19, n. 6, p. 741-760, 2006.

QUEIROZ, K. C.; ALFENAS, R. C. G.; SILVA, I. N. Hábitos alimentares e perfil de ingestão de energia e nutrientes de crianças e adolescentes com diabetes mellitus tipo 1. Revista Médica de Minas Gerais, 25(3), p. 330-337, 2015.

SANTOS, A. R. O. et al. Prevalência e fatores de proteção para o controle glicêmico em crianças portadoras de diabetes melito tipo 1. Revista de Ciências Médias e Biológicas, v. 15, n. 3, p. 317-322, 2016.
SOCIEDADE BRASILEIRA DE DIABETES. Diretrizes da Sociedade Brasileira de Diabetes. Aspectos gerais do tratamento de crianças e adolescentes com diabetes mellitus tipo 1. São Paulo, SP, 2017a.

SOCIEDADE BRASILEIRA DE DIABETES. Diretrizes da Sociedade Brasileira de Diabetes. Classificação e diagnóstico do diabetes mellitus. São Paulo, SP, 2017b.

SOCIEDADE BRASILEIRA DE DIABETES. Diretrizes da Sociedade Brasileira de Diabetes. Metas glicêmicas adultos, crianças e gestantes. São Paulo, SP, 2017c.

TELES, A. S.; FORNÉS, N. S. Consumo alimentar e controle metabólico em crianças e adolescentes portadores de diabetes melito tipo 1. Revista Paulista de Pediatria, v. 29, n. 3, p. 378-384, 2011.

WYLIE-ROSETT, J. et al. Medical nutrition therapy for youth with type 1 diabetes mellitus: more than carbohydrate counting. Journal of Academic of Nutrition and Dietetics, $\mathrm{p}$. 1.724-1.727, 2012. 